\title{
Corrigendum
}

\section{Determination of adsorption-controlled growth windows of chalcogenide perovskites - CORRIGENDUM}

\author{
Stephen A. Filippone, Yi-Yang Sun, and R. Jaramillo
}

doi.org/10.1557/mrc.2018.10, Published by Materials Research Society with Cambridge University Press, February 12,2018

In the original publication of Filippone et al. ${ }^{[1]}$ a number of errors were introduced after a numerical error in the calculated formation energies of the $\mathrm{Zr}$ - and Hf-containing materials that were studied. All corrections are listed below.

Caption of Table 1, "eV/formula unit" should be "eV/unit cell."

Page 3, right column, paragraph 2, line 8: Temperature range given as " $1300-1500{ }^{\circ} \mathrm{C}$ " should be " $1300-1350{ }^{\circ} \mathrm{C}$."

Figures 1 and 2 have been corrected and are reproduced here. The caption to Figure 2 should additionally include the sentence, "The effect of such errors is very small, and the red lines overlap the underlying black line in the plot."

Page 5, left column, paragraph 1, line 7: " $\mathrm{ZrSrS}_{3}$ " should be deleted so that the sentence reads, "Using for example $1000{ }^{\circ} \mathrm{C}, \mathrm{BaZrS}{ }_{3}$, $\mathrm{BaHfS}_{3}$, and $\mathrm{SrHfH}_{3}$ require a maximum pressure of about $10^{-9}$ torr for adsorption-limited growth."

Page 5, left column, paragraph 1, lines 10-13: The two sentences beginning with, "BaTiS 3 does not form. .." and ending with "...stable solid phase TiS," should say, "BaTiS ${ }_{3}$ and $\mathrm{SrZrS}_{3}$ do not have growth windows."

Page 5, left column, paragraph 2, lines 3-9: The last three sentences of the paragraph should be replaced with, "The growth windows for BaZrS 3 , $\mathrm{BaHfS}_{3}, \mathrm{BaZrSe}_{3}$, and $\mathrm{SrHfS}_{3}$ are all very narrow. The widest is that for $\mathrm{BaHfS}_{3}$, extending for one order of magnitude in pressure."

Page 5, right column, paragraph 1, line 11: “- -0.447 eV/f.u.” should be “ $-0.112 \mathrm{eV} /$ formula unit."

Page 6, left column, paragraph 3, lines 17-18: The last sentence should end after "negligible" so that the sentence reads, "The effect on the predicted growth window is negligible."

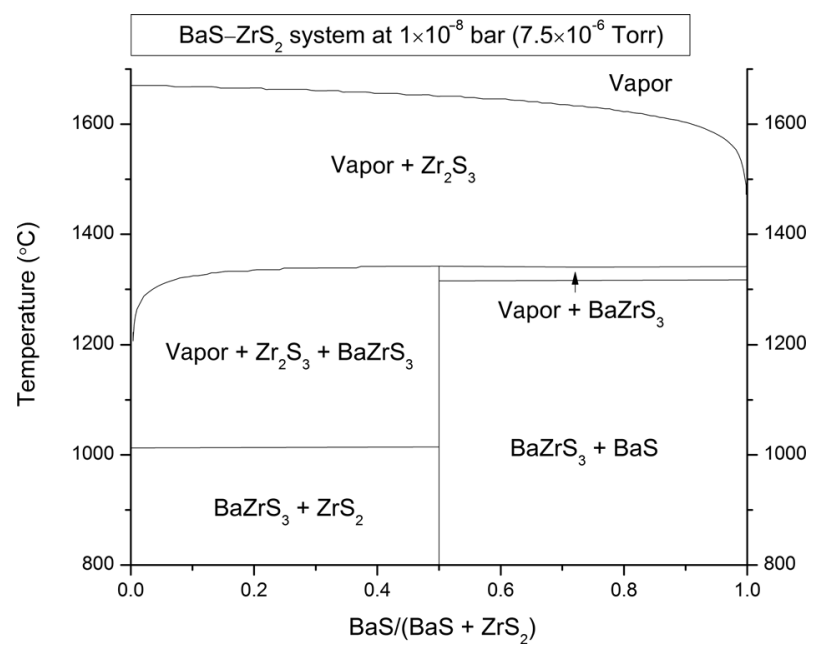

Figure 1 Composition-temperature equilibrium phase diagram for the pseudo-binary BaS-ZrS 2 system at a pressure of $7.5 \times 10^{-6}$ torr. The thermodynamic growth window for $\mathrm{BaZrS}_{3}$ is on the Ba-rich side of the phase diagram. 

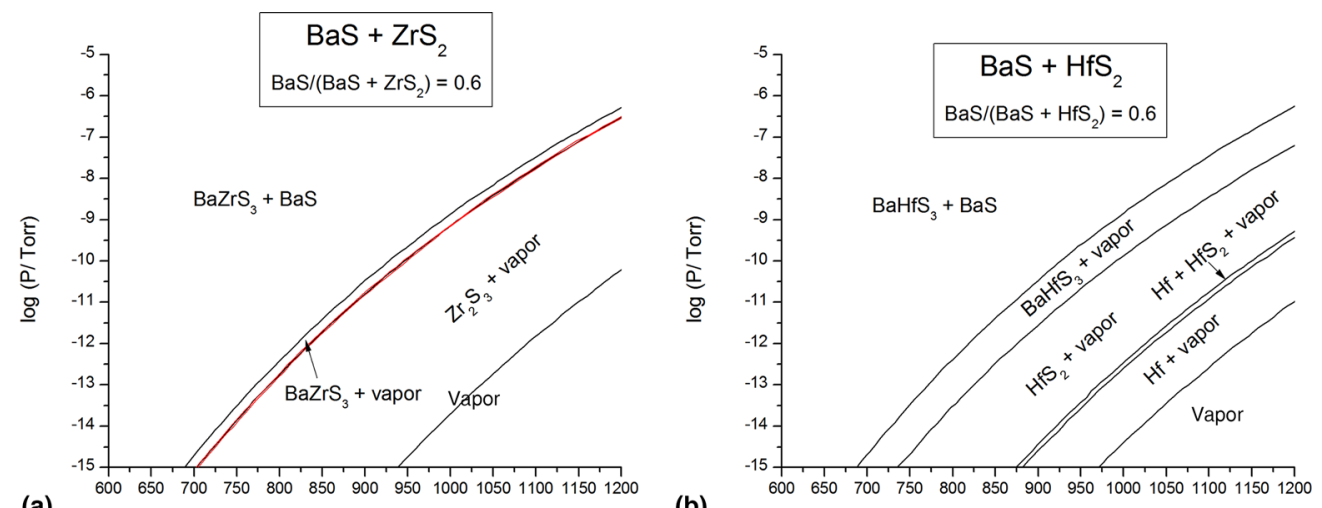

(a)

(b)
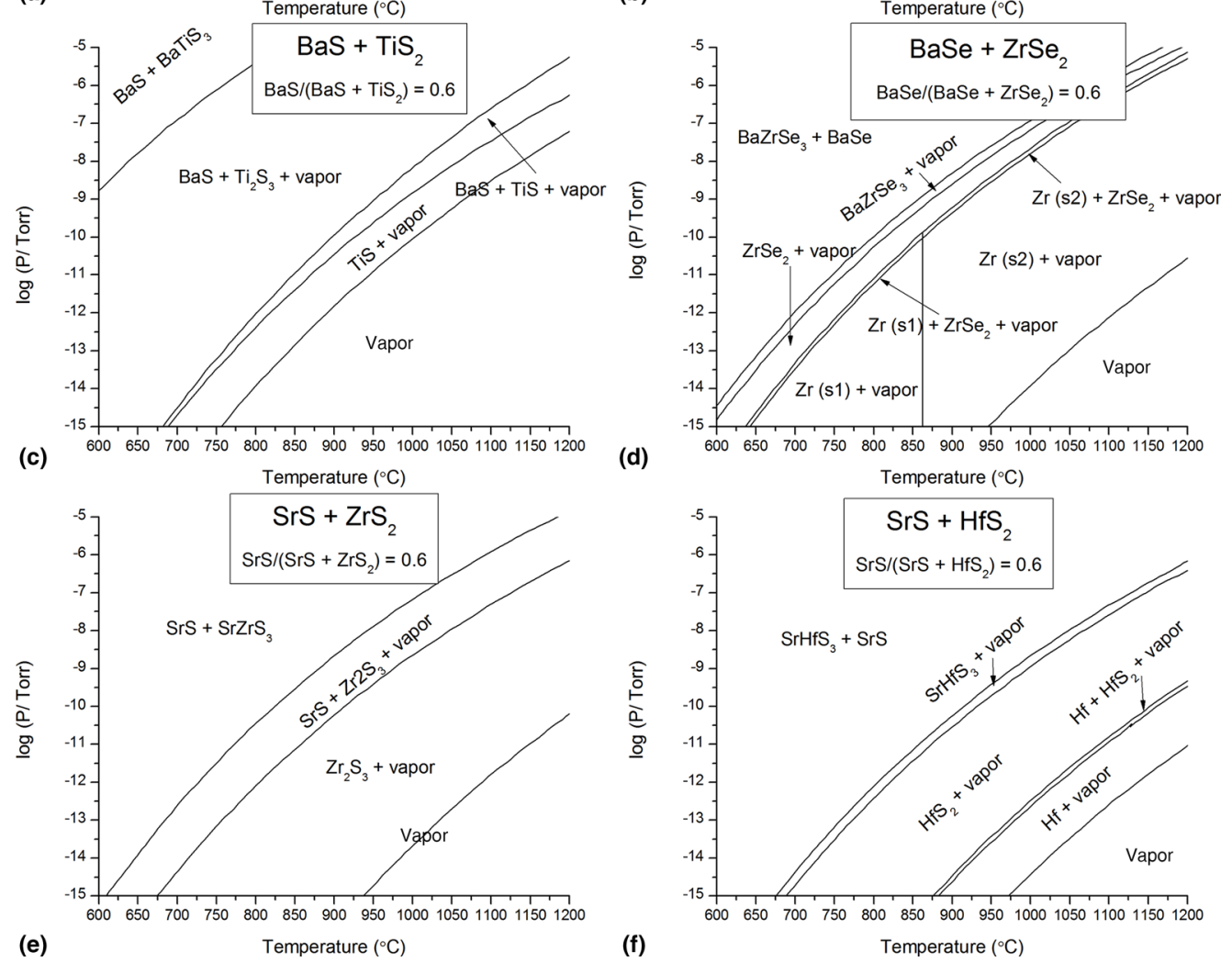

Figure 2 Temperature-pressure phase diagrams for six of chalcogenide perovskites that are calculated to be thermodynamically stable relative to decomposition into binary chalcogenides. The fixed composition in each case is a mole fraction of 0.6 of the ACh component. In each case, the thermodynamic growth window is labeled " $\mathrm{ABCh} \mathrm{H}_{3}$ + vapor", for variable elements $\mathrm{A}, \mathrm{B}$, and $\mathrm{Ch}$. Plots are ordered by the formation energy of the ternary phase from the binaries, from most to least stable. (a) BaS-ZrS 2 , (b) BaS-HfS ${ }_{2}$, (c) BaS- $\mathrm{TiS}_{2}$, (d) $\mathrm{BaSe}_{\mathrm{Z}} \mathrm{ZrSe}_{2}$, (e) $\mathrm{SrS}-\mathrm{HfS}_{2}$, (f) $\mathrm{SrS}-\mathrm{ZrS}$. The additional red lines in (a) show how the lower limit of the growth window would be changed by a $\pm 5 \%$ error in the formation energy of $\mathrm{BaZrS}_{3}$. The effect of such errors is very small, and the red lines overlap the underlying black line in the plot.

Page 6, right column, paragraph 1, lines 6-9: The sentence beginning, "The growth window for..." should be replaced with "As a rule, the growth windows for selenides may be more accessible than those for sulfides."

The authors apologize for these errors. The message and conclusions of the paper remain unchanged. The authors predict that chalcogenide perovskites containing transition metals may be very difficult to grow by the adsorption-controlled technique, but those containing main group metals may be quite accessible.

\section{Reference}

1.S.A. Filipone, Y.-Y. Sun, and R. Jaramillo: Determination of adsorption-controlled growth windows of chalcogenide perovskites. MRS Commun. 8, 145-151 (2018). 